\title{
Rapid detection of papillary thyroid carcinoma by fluorescence imaging using a $\gamma$-glutamyltranspeptidase-specific probe: a pilot study
}

Rumi Hino ${ }^{1,2^{*}}$ D, Naoko Inoshita ${ }^{2}$, Toyoki Yoshimoto ${ }^{2}$, Makiko Ogawa $^{2}$, Daishu Miura ${ }^{3}$, Ryoko Watanabe ${ }^{4}$, Kenta Watanabe ${ }^{4}$, Mako Kamiya ${ }^{5,6}$ and Yasteru Urano ${ }^{5,7,8}$

\begin{abstract}
Background: Nodular lesions of the thyroid gland, including papillary thyroid carcinoma (PTC), may be difficult to diagnose by imaging, such as in ultrasonic echo testing, or by needle biopsy. Definitive diagnosis is made by pathological examination but takes several days. A more rapid and simple method to clarify whether thyroid nodular lesions are benign or malignant is needed. Fluorescence imaging with $\gamma$-glutamyl hydroxymethyl rhodamine green (gGlu-HMRG) uses $\gamma$-glutamyltranspeptidase (GGT), a cell-surface enzyme, to hydrolyze the $\gamma$-glutamyl peptide and transfer the $\gamma$-glutamyl group. GGT is overexpressed in several cancers, such as breast, lung, and liver cancers. This imaging method is rapid and useful for detecting such cancers. In this study, we tried to develop a rapid fluorescence detection method for clinical samples of thyroid cancer, especially papillary carcinoma.

Methods: Fluorescence imaging with gGlu-HMRG was performed to detect PTC using 23 surgically resected clinical samples. A portable imaging device conveniently captured white-light images and fluorescence images with blue excitation light. Hematoxylin-eosin (HE) staining was used to evaluate which fluorescent regions coincided with cancer, and immunohistochemical examination was used to detect GGT expression.

Results: All 16 PTC samples exhibited fluorescence after topical application of gGlu-HMRG, whereas the normal sections of each sample showed no fluorescence. HE staining revealed that each fluorescent region corresponded to a region with carcinoma. The PTC samples also exhibited GGT expression, as confirmed by immunohistochemistry.
\end{abstract}

Conclusions: All PTC samples were detected by fluorescence imaging with gGlu-HMRG. Thus, fluorescence imaging with gGlu-HMRG is a rapid, simple, and powerful detection tool for PTC.

Keywords: Thyroid cancer, Papillary thyroid carcinoma, Fluorescence imaging, y-glutamyltranspeptidase, y-glutamyl hydroxymethyl rhodamine green

\footnotetext{
* Correspondence: rumih0301@ic.daito.ac.jp

${ }^{1}$ Department of Sports and Health Science, Daito Bunka University, 560 Iwadono, Higashimathuyama-shi, Saitama 355-8501, Japan

${ }^{2}$ Department of Pathology, Toranomon Hospital, Tokyo 105-0001, Japan

Full list of author information is available at the end of the article
}

(c) The Author(s). 2018 Open Access This article is distributed under the terms of the Creative Commons Attribution 4.0 International License (http://creativecommons.org/licenses/by/4.0/) which permits unrestricted use, distribution, and reproduction in any medium, provided you give appropriate credit to the original author(s) and the source, provide a link to the Creative Commons license, and indicate if changes were made. The Creative Commons Public Domain Dedication waiver (http://creativecommons.org/publicdomain/zero/1.0/) applies to the data made available in this article, unless otherwise stated. 


\section{Background}

Papillary thyroid carcinoma (PTC) is the most common thyroid cancer, comprising $70-80 \%$ of all thyroid cancers [1]. Although most PTC cases have good prognoses, there is a high-risk group comprising patients with distant metastasis and older patients ( $>50$ years of age) accompanied with massive extra-thyroidal invasion or large lymph node metastasis $(>3.0 \mathrm{~cm})$, and the case-specific death was reported to be $20 \%(44 / 220$ cases) [2]. Patients younger than 16 years with PTC, node metastasis $>3.0 \mathrm{~cm}$, and significant extension are reported to have poor prognoses [3]. However, nodular lesions of the thyroid gland, including PTC, may be difficult to diagnose by imaging, such as in ultrasonic echo testing, or by needle biopsy. In differential diagnosis, malignant tumors, benign tumors, and non-neoplastic lesions are predicted. Definitive diagnosis is made by pathological examination, but this takes a long time, i.e., at least 4 days, and requires multiple laboratory technicians because of steps such as processing, fixation, embedding, sectioning, and staining. The more quickly nodular lesions are diagnosed, the earlier the treatment may begin. Furthermore, simplicity of diagnosis is desired, that is, malignant diagnosis should be able to be determined even if there is no laboratory technician or pathologist. These aspects are of particular importance in cases of malignant tumors.

$\gamma$-Glutamyltranspeptidase (GGT) is a cell surface enzyme that cleaves glutathione, hydrolyzes the $\gamma$-glutamyl peptide, and transfers the $\gamma$-glutamyl group. $\gamma$-Glutamyl hydroxymethyl rhodamine green (gGlu-HMRG) is a nonfluorescent dye that is converted to highly fluorescent hydroxymethyl rhodamine green (HMRG) upon reaction with GGT [2]. GGT is purportedly overexpressed in several human cancers, such as breast, ovarian, brain, lung, colon, and peritoneum cancers, as well as head and neck squamous cell carcinoma, and methods exploiting gGlu-HMRG and GGT have been applied to detect such cancers [4-11]. When colorless gGlu-HMRG is sprayed on surgically resected breast cancer tissue, the cancer cells exhibit green fluorescence 1-2 min afterwards [12]. The brightness of the fluorescent agent is grossly confirmed, and green fluorescence indicates the presence of cancer [12]. Until the development of this method, there was no technique capable of visualizing cancer during surgery in such a short time; furthermore, this detection method using gGlu-HMRG is reported to be very simple $[4,7,9]$. No study has examined the use of the GGT-gGlu-HMRG detection method on primary human thyroid tumors.

In this study, we examined whether thyroid lesions were distinguishable as benign or malignant by the GGT-gGlu-HMRG method during surgical procedures.

\section{Methods}

\section{Fluorescent probe}

gGlu-HMRG, a fluorescent probe targeting GGT, was synthesized as previously described ${ }^{2}$. It was resuspended in $10 \mathrm{mM}$ dimethyl sulfoxide (DMSO, Sigma-Aldrich, St. Louis, MO, USA) and restored. The stock solution of gGlu-HMRG was diluted to the final concentration, $100 \mu \mathrm{M}$ or $1 \mathrm{mM}$, in phosphate-buffered saline (PBS, Wako Pure Chemical Industries, Tokyo, Japan).

\section{Study of patient specimens}

Twenty-three cases of thyroid lesions were examined. All samples were surgically resected by the Department of Breast and Endocrine Surgery or Otorhinolaryngology, Toranomon Hospital, between 2014 and 2017.

The resected specimens were immediately extracted, and approximately $5 \mathrm{~mm}$ [5] of the thyroid lesions and normal sites was also excised. Fluorescence imaging was performed using a fluorescence imaging system (Discovery; INDEC Medical Systems, Santa Clara, CA, USA). White-light images of specimens with no fluorescent probe were captured first, and then fluorescence images through yellow emission filter with $450-490 \mathrm{~nm}$ blue excitation light were captured after $100 \mu \mathrm{M}$ gGlu-HMRG was sprayed on both the lesion and normal tissue segments of the specimens. Fluorescence images were captured $0,0.5,1,2,3,5,7$, and 10 min after gGlu-HMRG spraying. Subsequently, the specimens were fixed with formalin and subjected to hematoxylin-eosin (HE) staining.

Histopathology by HE and immunohistochemical staining HE staining was performed to confirm the pathological findings. Specimens were fixed, embedded in paraffin, and cut into $3-\mu \mathrm{m}$ sections prior to staining with HE. We determined which parts of tissues exhibited green or no fluorescent signals using a fluorescence imaging system with a microscope (Nikon). In the case of tumors, histological type, capsular invasion, and lymph-vascular invasion were evaluated from sections of each sample according to the World Health Organization classification scheme.

Immunohistochemical analysis of GGT1 was performed using an auto-immunostainer, BOND III (Leica, Wetzlar, Germany), with antibodies against GGT1 (mouse.

monoclonal, dilution 1: 600; Abcam, Cambridge, UK). HepG2 cells were used as a positive control. Higher than $10 \%$ positivity among tumor cells was considered positive staining.

\section{Cell lines, cell culture, and cell block construction}

Two cell lines were prepared with high and low GGT expression. The high GGT expression cell line was 
A549, established from human lung adenocarcinoma and reported as a positive control for gGlu-HMRG [9], and the low GGT expression cell lone was SW782, a noncancerous line of human pre-adipocytes.

HepG2 cells were cultured in Dulbecco's modified Eagle's medium (DMEM; Nacalai Tesque, Kyoto, Japan) supplemented with $10 \%$ fetal bovine serum (FBS; Life Technologies, Carlsbad, CA, USA), $100 \mathrm{U} / \mathrm{mL}$ penicillin, and $100 \mu \mathrm{g} / \mathrm{mL}$ streptomycin (Wako).

Cell blocks were constructed as follows. HepG2 cells were cultured in 100-mm plates (Nacalai Tesque) and harvested using a scraper (Nacalai Tesque). Cells in $200 \mu \mathrm{L}$ of saline were then centrifuged at $3000 \mathrm{rpm}$ for 5 min, the supernatant was discarded, and the cells were fixed in formaldehyde saline overnight. Fixed cells were embedded in agarose gel. The cell blocks were cut into thin sections for $\mathrm{HE}$ and immunohistochemical staining.

\section{Results}

Confirmation of the function of the fluorescent probe

To examine the function of the fluorescent probe, two cell lines, aA549 and SW782, were used. A549 cells exhibited bright green fluorescence on detection of gGlu-HMRG, and we determined that they were suitable as a positive control in this study (Fig. 1). SW782, a noncancerous cell line of human pre-adipocytes, showed no fluorescence using the gGlu-HMRG probe method and was suitable as a negative control (Fig. 1).
Imaging of specimens from patients with thyroid cancer The ability of gGlu-HMRG to detect malignant thyroid cancer was examined using surgically resected thyroid lesion samples from 23 cases. Information related to the 23 cases is documented in Table 1. Representative images of a human thyroid tumor and normal tissue taken using the fluorescence imaging system (Discovery) are shown in Fig. 2. White-light images of the tumor and normal tissue were taken first (Fig. 2a, c), and then fluorescence images were obtained using gGlu-HMRG (Fig. 2b, d). All cases were evaluated by comparison with normal tissue. Thyroid lesions in this study consisted of 16 PTCs, 3 adenomatous goiters, 1 Graves' disease, 1 follicular adenoma, and 1 thyroiditis.

After treatment with gGlu-HMRG, all 16 PTC samples (Figs. 1 and 2; Case 2), including those from recurrence cases (Fig. 3; Case 3), exhibited green fluorescence, whereas normal tissue from the same cases showed no fluorescence. Among the thyroid samples, those from the follicular adenoma, Graves' disease, thyroiditis (Fig. 3; Case 5), and 3 adenomatous goiter cases showed no fluorescence. One of the 3 adenomatous goiter cases was a mediastinal goiter (Fig. 3; Case 4).

\section{Pathological confirmation of thyroid lesions}

After fluorescence imaging, all samples were embedded in paraffin blocks, and each tissue was pathologically confirmed by HE staining. Of the 23 cases studied, 19

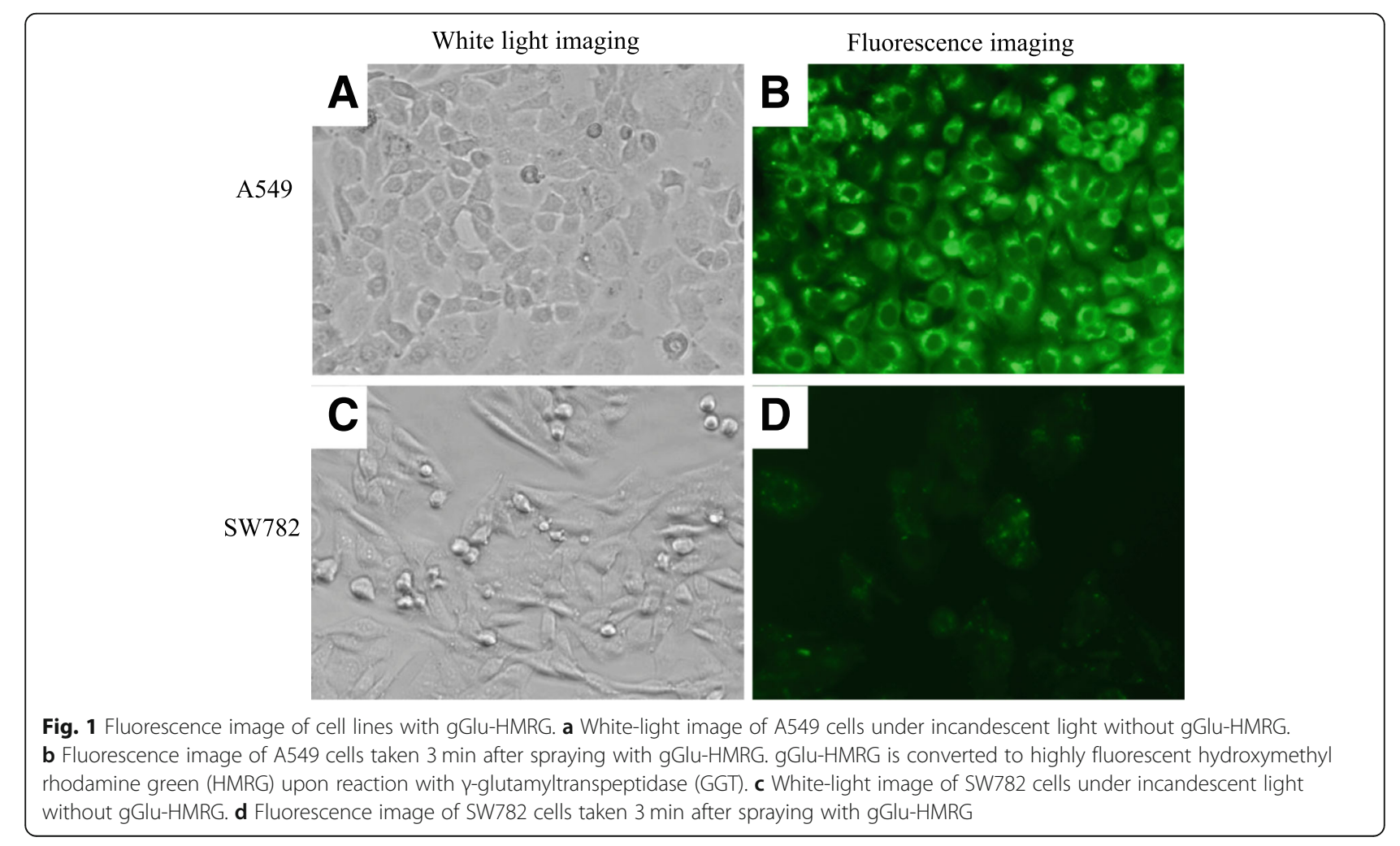


Table 1 Summary of data in patients with thyroid lesion

\begin{tabular}{|c|c|c|c|c|c|c|}
\hline Patient & Age & Sex & Preoperative clinical diagnosis & Pathological diagnosis & gGlu-HMR & ormal \\
\hline 1 & 30 & $\mathrm{~F}$ & PTC $^{* b}$ & PTC & positive & negative \\
\hline 2 & 32 & $\mathrm{~F}$ & PTC & PTC & positive & negative \\
\hline 3 & 46 & $\mathrm{~F}$ & PTC (recurrence) & PTC(recurrence) & positive & negative \\
\hline 4 & 55 & $\mathrm{~F}$ & Thyroid malignant tumor & Mediastinal goiter & negative & negative \\
\hline 5 & 78 & M & Lymphoma & Inflammation & negative & negative \\
\hline 6 & 43 & $\mathrm{~F}$ & PTC & PTC & positive & negative \\
\hline 7 & 47 & $\mathrm{~F}$ & Thyroid tumor & Follicular adenoma & negative & negative \\
\hline 8 & 47 & $\mathrm{~F}$ & PTC & PTC & positive & negative \\
\hline 9 & 44 & $\mathrm{~F}$ & PTC & PTC & positive & negative \\
\hline 10 & 61 & $\mathrm{~F}$ & PTC & PTC & positive & negative \\
\hline 11 & 32 & $\mathrm{~F}$ & PTC & PTC & positive & negative \\
\hline 12 & 48 & M & PTC & PTC & positive & negative \\
\hline 13 & 23 & $\mathrm{~F}$ & Thyroid tumor & Goiter ${ }^{*} c$ & negative & negative \\
\hline 14 & 44 & M & PTC & PTC & positive & negative \\
\hline 15 & 58 & $\mathrm{~F}$ & Thyroid tumor & Goiter & negative & negative \\
\hline 16 & 72 & $\mathrm{~F}$ & PTC & PTC & positive & negative \\
\hline 17 & 57 & M & PTC & PTC & positive & negative \\
\hline 18 & 68 & $\mathrm{~F}$ & Thyroid lesion & Graves ${ }^{*}{ }^{* d}$ & negative & negative \\
\hline 19 & 71 & $\mathrm{~F}$ & PTC & PTC & negative & negative \\
\hline 20 & 55 & $\mathrm{~F}$ & PTC & PTC & positive & negative \\
\hline 21 & 52 & $\mathrm{~F}$ & PTC & PTC & positive & negative \\
\hline 22 & 69 & M & Goiter & Goiter & negative & negative \\
\hline 23 & 64 & M & PTC & PTC & positive & negative \\
\hline
\end{tabular}

${ }^{{ }^{a}}$ gGGlu-HMRG: detection results by using $\mathrm{Y}$ - glutamyl hydroxymethyl rhodamine green, ${ }^{\text {*b } P T C: ~ P a p i l l a r y ~ t h y r o i d ~ c a r c i n o m a, ~}$

${ }^{*}$ 'Goiter: Adenomatous goiter, ${ }^{* d}$ Graves': Graves' disease or Basedow disease

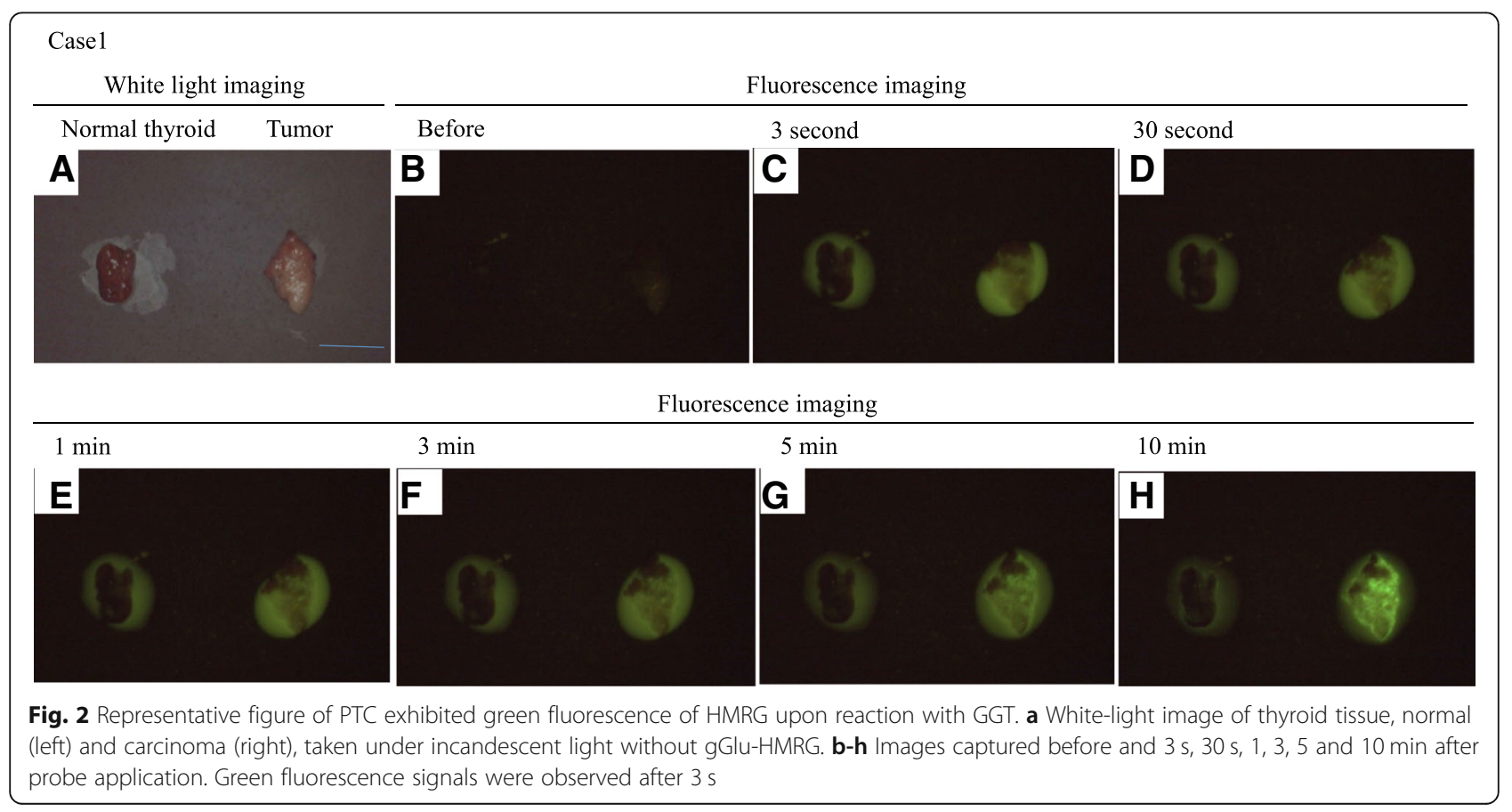




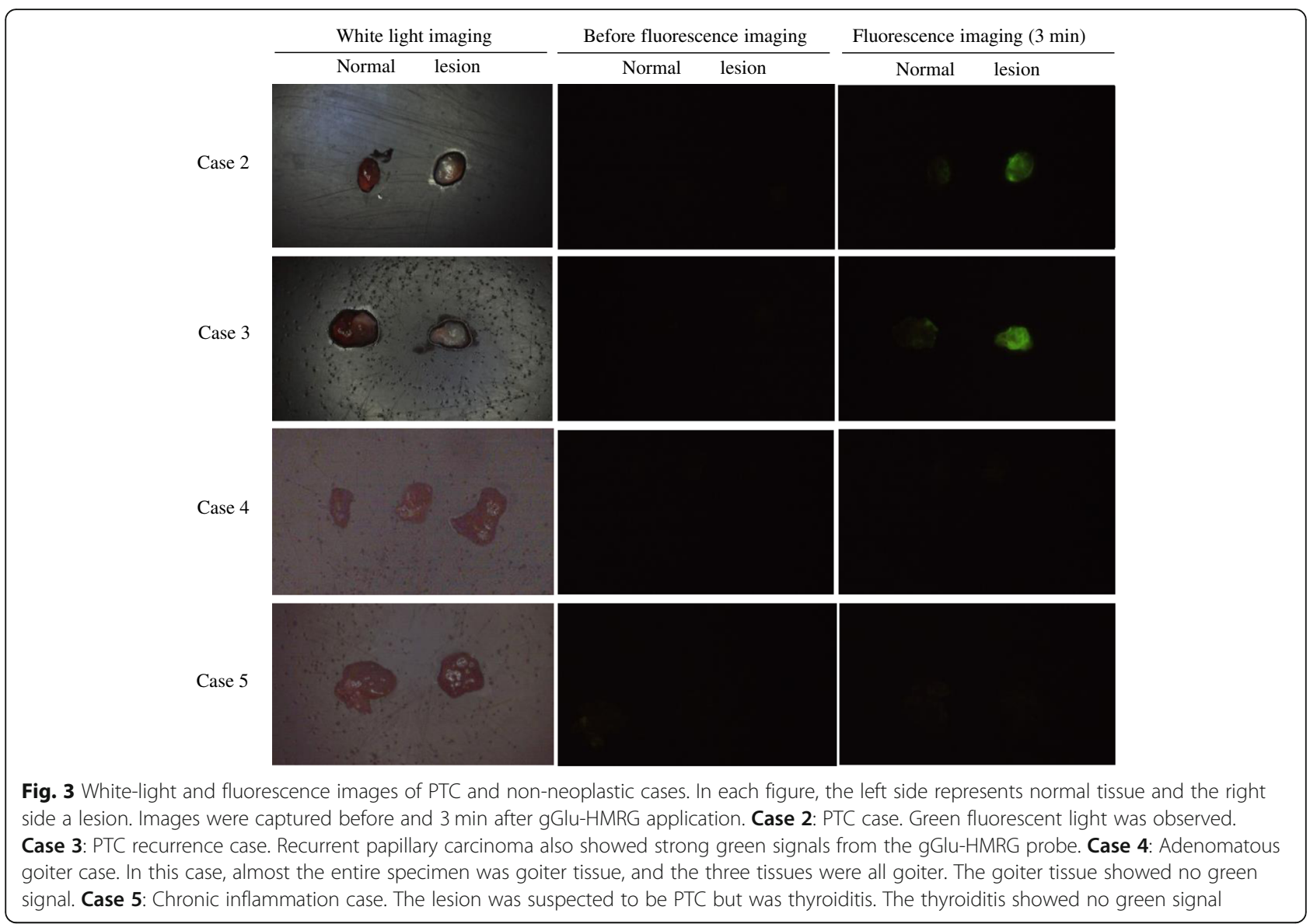

were papillary carcinoma (Fig. 4; Case 1 and 2), 3 were adenomatous goiter (Fig. 4; Case 4), 1 was follicular adenoma, and 1 was thyroiditis (Fig. 2; Case 5). Two pathologists confirmed the diagnoses. All thyroid lesions were confirmed by HE staining.

\section{Evaluation of GGT expression by immunostaining with anti-GGT1 antibody}

To confirm the expression of GGT in all samples, immunohistochemical examination was performed. HepG2 and A549 cells were used as positive controls for GGT immunostaining, whereas SW782 cells were used as a negative control. We conducted GGT1 immunostaining of all 23 specimens used in fluorescence imaging. Representative staining of a thyroid tumor is shown in Fig. 5. Positive staining was seen in thyroid papillary carcinoma tissue (14/16 cases) (Fig. 5a). No GGT1 expression was observed in any normal thyroid tissue (16/16 cases) (Fig. 5c). Samples from the thyroiditis, follicular adenoma, Graves' disease, and 3 adenomatous goiter cases including 1 mediastinal goiter case were judged negative by GGT1 immunohistochemical staining.

\section{Discussion}

As GGT is overexpressed on the membranes of various cancer cells, gGlu-HMRG has been reported as a tool to detect breast cancer [12], lung cancer [9], colorectal tumors [10], head and neck squamous cell carcinoma [7], and prostate cancer [13]. The sensitivity and specificity of gGlu-HMRG in lung cancer detection are 43.8 and $84.9 \%$, respectively [9]. gGlu-HMRG also reportedly enabled rapid fluorescence imaging of 54 and $76 \%$ of colorectal adenomas and carcinomas, respectively [10]. In a report on 8 head and neck tumor lesions, all samples exhibited fluorescence after application of gGlu-HMRG [7]. The sensitivity and specificity of breast cancer detection by gGlu-HMRG are 92 and $94 \%$, respectively [12]. GGT is reportedly highly expressed in malignant ovarian tumors, breast cancer, carcinoma of the thyroid, and lung cancer [14-16]; however, whether fluorescence imaging using gGlu-HMRG can detect human thyroid cancer remains to be elucidated. This study is the first report demonstrating the efficacy of gGlu-HMRG in the detection of thyroid cancer.

In this study, we evaluated the detection of thyroid cancer, especially PTC, by gGlu-HMRG. All PTCs were detected after spraying with gGlu-HMRG, and 


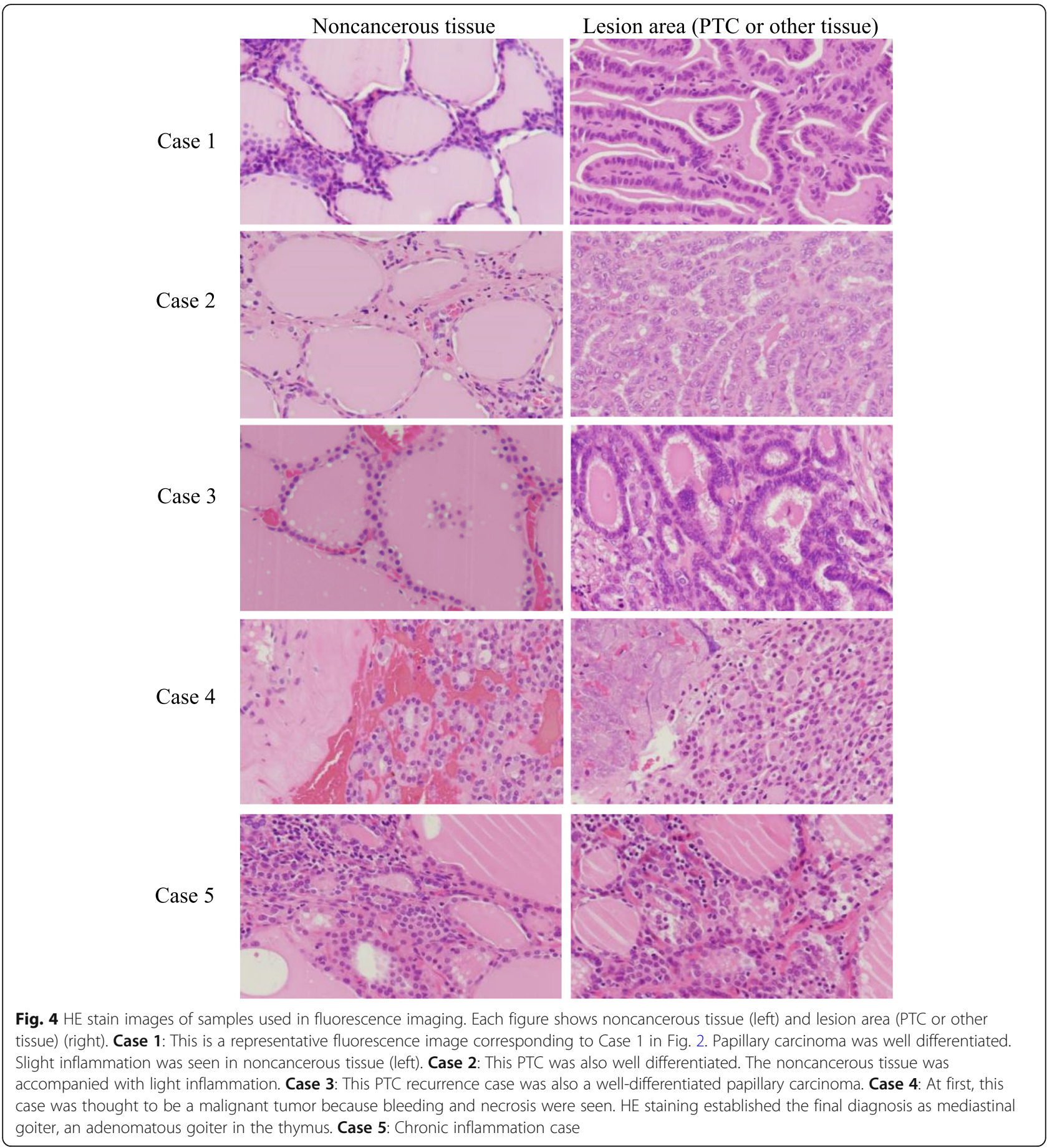

fluorescence images were obtained (16/16 cases, $100 \%)$; in contrast, normal tissue counterparts from the same cases exhibited no fluorescent signals $(0 / 16$ cases, $0 \%)$. As only 16 cases were studied, evaluation of sensitivity and specificity is difficult, but both are expected to be higher than 95\%. Future clinical trials studying larger numbers of PTC cases would help to clarify these concerns. Additionally, the cases of adenomatous goiter, thyroiditis, Graves' disease, and follicular adenoma showed no fluorescent signals after gGlu-HMRG application. These results indicate that the gGlu-HMRG method is useful for the specific detection of PTC and that the method is easy and fast when used on surgically resected specimens.

Frozen-section diagnosis is performed during surgical operation. The process involves preparation of frozen 


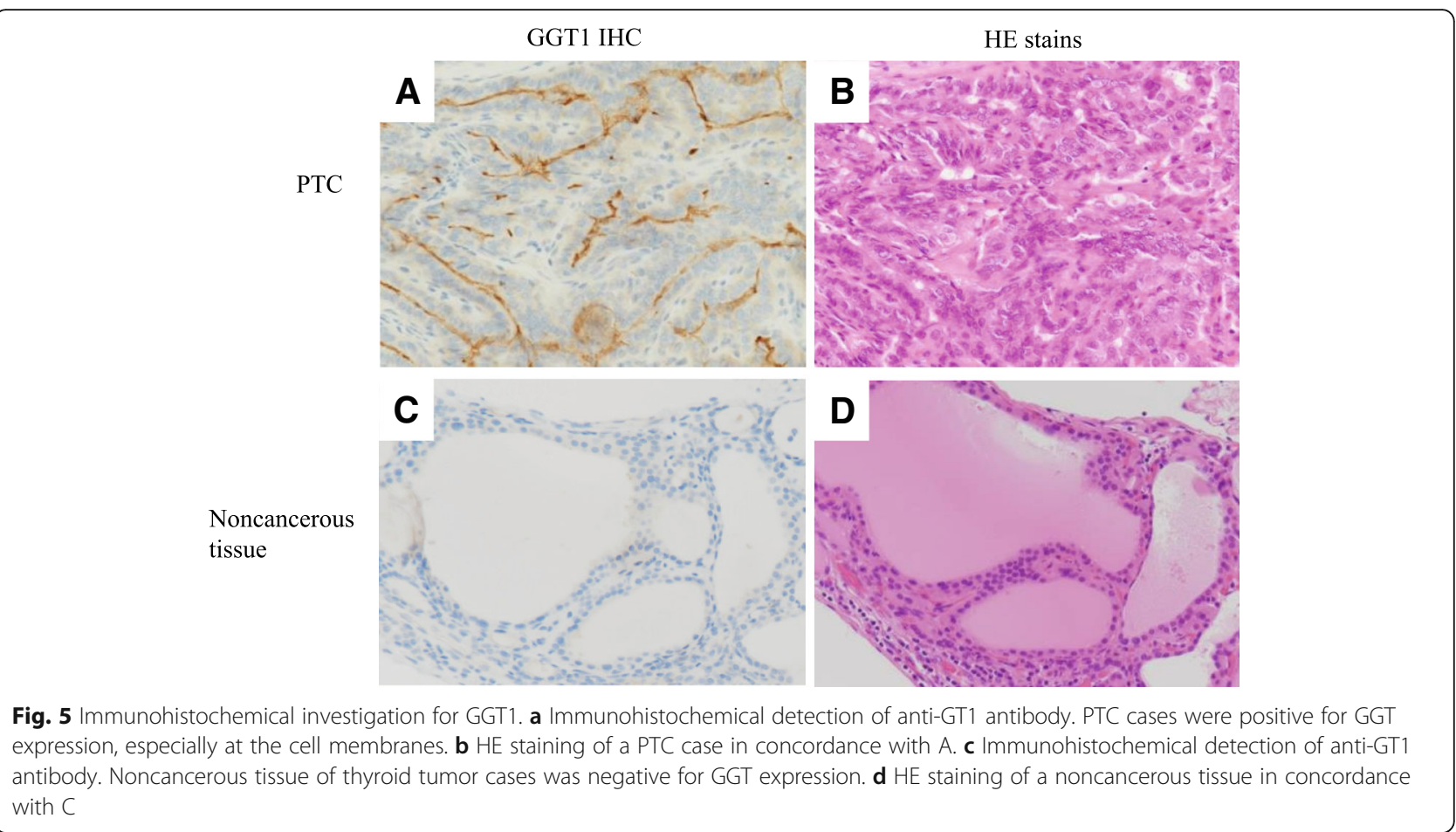

sections, HE staining, and diagnosis by a pathologist and takes $15-20 \mathrm{~min}$. It has been a useful method for judging whether tissue is malignant during an operation. However, the gGlu-HMRG detection method requires much less time than frozen-section diagnosis, taking only $2-3$ min. Tumor lesions become fluorescent within a few minutes of gGlu-HMRG application. The judgment is very simple; green fluorescence indicates the presence of a malignant tumor, whereas benign or non-neoplastic lesions show no fluorescence. Frozen-section diagnosis requires several years of pathological training, whereas recognition of green fluorescence requires no training. Therefore, gGlu-HMRG detection of malignant tumors is simpler than frozen-section diagnosis, and it could be applied in hospitals without laboratory technicians or pathologists.

There were 2 interesting cases in this experiment. The first was a mediastinal goiter case. When the specimen was carried to the pathology laboratory from the operating room, a malignancy was strongly suspected because the tissue exhibited massive bleeding and necrosis. Spraying with gGlu-HMRG resulted in no fluorescent green signal, suggesting no malignancy. The final pathological diagnosis made by HE staining was mediastinal goiter, which is neither a tumor nor malignant, but rather an enlarged thyroid gland. The second case was thyroiditis. The lesion was preoperatively expected to be PTC. Applying gGlu-HMRG to the sample resulted in no green signal. HE staining revealed that the lesion was not PTC, but thyroiditis. Both cases demonstrated that the gGlu-HMRG method was capable of correctly judging malignancy. In both cases, PTC was suspected, but the fluorescent probe showed that the lesions were not PTC before pathological diagnosis.

Immunohistochemical examination of cancer and normal tissue was used to validate GGT expression. GGT1 is reportedly expressed in the cell membrane, and GGT is a membrane-bound enzyme involved in the metabolism of glutathione ( $\gamma$-glutamyl-cysteinyl-glycine; GSH) [17]. Studies have suggested that GGT plays an important role in tumor development, progression, invasion, drug resistance, and prognosis [17-22]. Small tumors are reportedly rapidly detectable by fluorescence imaging in vivo [23-28]. Several studies have reported that GGT is highly expressed in cancer cell membranes, as determined by immunohistochemical methods [7, 9]. In this study, 14 thyroid cancer samples among 16 cases were positive for GGT in the cell membranes; in contrast, cases involving non-cancerous or inflammatory lesions were negative. The negativity of the two cancer cases for GGT1 antibody might be attributable to the low specificity of the GGT antibody to the GGT1 antigen. However, all the cancer tissues were confirmed pathologically according to the parts exhibiting fluorescence. The results of immunostaining also supported the pathological diagnosis. These results showed that GGT is expressed in PTC and that the gGlu-HMRG method depends on GGT. 
In this study, we showed that the GGT fluorescent probe could be applied to thyroid cancer detection, especially PTC. This is the first time that thyroid cancer detection by the fluorescent probe has been demonstrated. We began the examination using PTC because we had access to more papillary carcinoma than other thyroid cancer samples. As a next step, we plan to perform a similar experiment for other thyroid carcinomas, such as follicular carcinoma.

\section{Conclusions}

In this study, all PTC samples exhibited green fluorescence after application of gGlu-HMRG, whereas normal tissue showed none. Furthermore, samples from cases of other diseases, including thyroiditis, adenomatous goiters, Graves' disease, and follicular adenoma, exhibited no fluorescence. These results were supported by immunohistochemistry using an antibody against GGT1. The main characteristic of this gGlu-HMRG fluorescence detection method is its specific detection of PTC. Thus, the gGlu-HMRG method is expected to be a very effective method for pathologically and clinically identifying PTC. A large clinical study of thyroid cancer to confirm the utility of this fluorescent probe method is warranted.

\section{Abbreviations}

DMEM: Dulbecco's modified Eagle's medium; DMSO: Dimethyl sulfoxide; FBS: Fetal bovine serum; gGlu-HMRG: Y-glutamyl hydroxymethyl rhodamine green; GGT: Y-glutamyltranspeptidase; GSH: Glutathione; HE: Hematoxylineosin; HMRG: Hydroxymethyl rhodamine green; PBS: Phosphate-buffered saline; PTC: Papillary thyroid carcinoma

\section{Acknowledgments}

We thank Mamoru lijima and Sayaka Ogikubo (Toranomon Hospital, Tokyo, Japan) for supporting pathological staining. This work was in part supported by Okinaka Memorial Institute for Medical Research.

\section{Funding}

Grant support: Grant-in aid for Scientific Research on Priority Areas from the Ministry of Education, Science, Sports, and Culture of Japan (17 K01824).

\section{Availability of data and materials}

The datasets used during the current study are available from the corresponding author on reasonable request.

\section{Authors' contributions}

All authors read and approved the final manuscript.

\section{Ethics approval and consent to participate}

The Toranomon Hospital ethics committee approved this study (recipient number: 936). We obtained informed consent from all patients.

\section{Consent for publication}

Written informed consent was obtained from the patients' guardian/parent/ next of kin for the publication of this report and any accompanying images.

\section{Competing interests}

The authors declare that they have no competing interests.

\section{Publisher's Note}

Springer Nature remains neutral with regard to jurisdictional claims in published maps and institutional affiliations.

\section{Author details}

${ }^{1}$ Department of Sports and Health Science, Daito Bunka University, 560 Iwadono, Higashimathuyama-shi, Saitama 355-8501, Japan. ${ }^{2}$ Department of Pathology, Toranomon Hospital, Tokyo 105-0001, Japan. ${ }^{3}$ Akasaka Miura Clinic, Tokyo 107-0052, Japan. ${ }^{4}$ Department of Otolaryngology, Toranomon Hospital, Tokyo 105-0001, Japan. ${ }^{5}$ Graduate School of Medicine, The University of Tokyo, Tokyo 113-0033, Japan. ' $\mathrm{PRESTO}$, Japan Science and Technology Agency (JST), Saitama 332-0012, Japan. ${ }^{7}$ Graduate School of Pharmaceutical Sciences, The University of Tokyo, Tokyo 113-0033, Japan. ${ }^{8}$ AMED CREST, Japan Agency for Medical Research and Development, Tokyo 100-0004, Japan.

Received: 15 August 2018 Accepted: 8 November 2018 Published online: 21 November 2018

\section{References}

1. Gonzalez-Gonzalez R, Bologna-Molina R, Carreon-Burciaga RG, Gomezpalacio-Gastelum M, Molina-Frechero N, Salazar-Rodriguez S. Papillary thyroid carcinoma: differential diagnosis and prognostic values of its different variants: review of the literature. ISRN Oncol. 2011;2011:915925.

2. Urano Y, Sakabe M, Kosaka N, Ogawa M, Mitsunaga M, Asanuma D, et al. Rapid cancer detection by topically spraying a gammaglutamyltranspeptidase-activated fluorescent probe. Sci Transl Med. 2011;3:110-9.

3. Ito Y, Kihara M, Takamura Y, Kobayashi K, Miya A, Hirokawa M, et al. Prognosis and prognostic factors of papillary thyroid carcinoma in patients under 20 years. Endocrine. 2012;59:539-45.

4. Shinden Y, Ueo H, Tobo T, Gamachi A, Utou M, Komatsu H, et al. Rapid diagnosis of lymph node metastasis in breast cancer using a new fluorescent method with gamma-glutamyl hydroxymethyl rhodamine green. Sci Rep. 2016;6:2752-5.

5. Hanigan MH, Frierson HF Jr, Brown JE, Lovell MA, Taylor PT. Human ovarian tumors express gamma-glutamyl transpeptidase. Cancer Res. 1994;54:286-90.

6. Harada T, Nakamura Y, Sato K, Nagaya T, Choyke PL, Seto Y, et al. Surgical tissue handling methods to optimize ex vivo fluorescence with the activatable optical probe gamma-glutamyl hydroxymethyl rhodamine green. Contrast Media Mol Imaging. 2016;11:572-8.

7. Mizushima T, Ohnishi S, Shimizu Y, Kato M, Sakamoto N, Urano Y. Fluorescent imaging of superficial head and neck squamous cell carcinoma using a gamma-glutamyltranspeptidase-activated targeting agent: a pilot study. BMC Cancer. 2016;16:411.

8. Schafer C, Fels C, Brucke M, Holzhausen HJ, Bahn H, Wellman M, et al. Gamma-glutamyl transferase expression in higher-grade astrocytic glioma. Acta Oncol. 2001;40:529-35.

9. Hino H, Kamiya M, Kitano K, Mizuno K, Tanaka S, Nishiyama N, et al. Rapid cancer fluorescence imaging using a gamma-glutamyltranspeptidasespecific probe for primary lung cancer. Transl Oncol. 2016;9:203-10.

10. Sato C, Abe S, Saito Y, So Tsuruki E, Takamaru H, Makazu M, et al. A pilot study of fluorescent imaging of colorectal tumors using a gammaglutamyl-transpeptidase-activatable fluorescent probe. Digestion. 2015;91:70-6.

11. Nakamura Y, Harada T, Nagaya T, Sato K, Okuyama S, Choyke PL, et al. Dynamic fluorescent imaging with the activatable probe, gamma-glutamyl hydroxymethyl rhodamine green in the detection of peritoneal cancer metastases: overcoming the problem of dilution when using a sprayable optical probe. Oncotarget. 2016;7:51124-37.

12. Ueo H, Shinden Y, Kobayashi H, Ueo H, Urano Y, Mimori K. Rapid intraoperative visualization of breast lesions with gamma-glutamyl hydroxymethyl rhodamine green. Sci Rep. 2015;5:12080.

13. Yogo T, Umezawa K, Kamiya M, Hino R, Urano Y. Development of an activatable fluorescent probe for prostate cancer imaging. Bioconjug Chem. 2017;28:2069-76.

14. Pompella A, De Tata V, Paolicchi A, Zunino F. Expression of gammaglutamyltransferase in cancer cells and its significance in drug resistance. Biochem Pharmacol. 2006;71:231-8.

15. Hanigan MH, Frierson HF Jr, Swanson PE, De Young BR. Altered expression of gamma-glutamyl transpeptidase in human tumors. Hum Pathol. 1999;30:300-5.

16. Hanigan MH, Gallagher BC, Taylor PT Jr, Large MK. Inhibition of gammaglutamyl transpeptidase activity by acivicin in vivo protects the kidney from cisplatin-induced toxicity. Cancer Res. 1994;54:5925-9. 
17. Corti A, Franzini M, Paolicchi A, Pompella A. Gamma-glutamyltransferase of cancer cells at the crossroads of tumor progression, drug resistance and drug targeting. Anticancer Res. 2010;30:1169-81.

18. Wen YF, Yang XZ, Zeng LS, Peng HH, Huang WJ, Cai LM, et al. Prognostic impact of pretherapeutic gamma-glutamyltransferase on patients with nasopharyngeal carcinoma. PLoS One. 2017;12:e0172345.

19. Hanigan MH, Gallagher BC, Townsend DM, Gabarra V. Gamma-glutamyl transpeptidase accelerates tumor growth and increases the resistance of tumors to cisplatin in vivo. Carcinogenesis. 1999;20:553-9.

20. Pompella A, Corti A, Paolicchi A, Giommarelli C, Zunino F. Gammaglutamyltransferase, redox regulation and cancer drug resistance. Curr Opin Pharmacol. 2007;7:360-6.

21. Van Hemelrijck M, Jassem W, Walldius G, Fentiman IS, Hammar N, Lambe M, et al. Gamma-glutamyltransferase and risk of cancer in a cohort of 545,460 persons - the Swedish AMORIS study. Eur J Cancer. 2011;47:2033-41.

22. Matsuzaki H, Kamiya M, Iwatate RJ, Asanuma D, Watanabe T, Urano Y. Novel hexosaminidase-targeting fluorescence probe for visualizing human colorectal cancer. Bioconjug Chem. 2016;27:973-81.

23. Kamiya M, Urano Y. Rapid and sensitive fluorescent imaging of tiny tumors in vivo and in clinical specimens. Curr Opin Chem Biol. 2016;33:9-15.

24. Shimane T, Aizawa H, Koike T, Kamiya M, Urano Y, Kurita H. Oral cancer intraoperative detection by topically spraying a $\gamma$-glutamyl transpeptidaseactivated fluorescent probe. Oral Oncol. 2016;54:e16-8.

25. Mori K, Ishizawa T, Yamashita S, Kamiya M, Urano Y, Kokudo N. Intraoperative visualization of pancreatic juice leaking from the pancreatic stump in a swine model. Gastroenterology. 2015;149:1334-6.

26. Asanuma D, Sakabe M, Kamiya M, Yamamoto K, Hiratake J, Ogawa M, et al. Sensitive $\beta$-galactosidase-targeting fluorescence probe for visualizing small peritoneal metastatic tumours in vivo. Nat Commun. 2015;6:6463.

27. Miyata A, Ishizawa T, Kamiya M, Shimizu A, Kaneko J, ljichi H, et al. Photoacoustic tomography of human hepatic malignancies using intraoperative indocyanine green fluorescence imaging. PLoS One. 2014:9:e112667.

28. Fujii T, Kamiya M, Urano Y. In vivo imaging of intraperitoneally disseminated tumors in model mice by using activatable fluorescent small-molecular probes for activity of cathepsins. Bioconjug Chem. 2014;25:1838-46.

Ready to submit your research? Choose BMC and benefit from:

- fast, convenient online submission

- thorough peer review by experienced researchers in your field

- rapid publication on acceptance

- support for research data, including large and complex data types

- gold Open Access which fosters wider collaboration and increased citations

- maximum visibility for your research: over $100 \mathrm{M}$ website views per year

At BMC, research is always in progress.

Learn more biomedcentral.com/submissions 